\title{
EXPERIMENTOS REPLICATIVOS DE GRABADOS EN PIEDRA. IMPLICANCIAS EN EL ARTE RUPESTRE DE LA LOCALIDAD ARQUEOLÓGICA DE PIEDRA MUSEO (SANTA CRUZ, ARGENTINA)
} STONE ENGRAVING REPLICATION EXPERIMENTS AND THEIR IMPLICATIONS
FORTHEROCK ART OFPIEDRA MUSEO (SANTA CRUZ, ARGENTINA)

\author{
ROCIO V. BLANCO* \& VIRGINIA LYNCH**
}

\begin{abstract}
Este trabajo presenta los avances en las investigaciones sobre las técnicas de producción de grabados rupestres de la localidad arqueológica Piedra Museo, Santa Cruz (Argentina). El afloramiento sobre el que se documentaron los petroglifos es producto de una ingresión marina del Terciario -de base coquinoide- sumamente friable, que pudo haber influenciado la toma de decisiones para realizar los motivos. Los objetivos formulados han sido caracterizar las técnicas de grabado empleadas en la localidad de estudio a partir de la experimentación y describir las trazas de uso dejadas en los instrumentos utilizados. Los resultados obtenidos permiten hacer una primera caracterización de los microrrastros sobre las piezas trabajadas, siendo coincidentes con los obtenidos por otros investigadores, más allá de las diferencias particulares. El análisis funcional junto con el estudio de las cadenas operativas implicadas en la producción de grabados, son etapas fundamentales para formular hipótesis referentes a las sociedades cazadoras-recolectoras del pasado.
\end{abstract}

Palabras clave: arte rupestre, Patagonia, análisis funcional, petroglifos, tecnología

In this paper we present advances in the research on engraving production techniques used at Piedra Museo archaeological locality, in Santa Cruz, Argentina. The outcrop on which the original engravings were documented resulted from a Tertiary marine transgression-coquinoid base-that is extremely friable, which could have influenced the decision to create these motifs. The goals of this paper are to describe the engraving techniques used at this archaeological site by carrying out experimental engraving and examining the traces left on the stone tools used for those experiments. The results allow us to identify the origin and development of micro-wear on the artefacts used, which is consistent with that observed by other researchers, with some particular differences. Both functional analysis and analysis of the "chaîne-opératoire" involved in petroglyph production are considered fundamental stages in the formulation of bypotheses about ancient bunter-gatherer societies.

Key words: rock art, Patagonia, functional analysis, petroglyphs, technology

\section{INTRODUCCIÓN}

El arte rupestre en la meseta Central de Santa Cruz ha sido investigado desde principios de la década de 1930 (Aparicio 1935), de manera intermitente en sus comienzos (Fiore \& Hernández Llosas 2007, para mayor detalle) y más regularmente a partir de la década de 1990 (Carden 2008a). En la actualidad se cuenta con un corpus considerable de datos, los cuales han sido tratados desde diferentes lineamientos teóricos. Las primeras investigaciones hicieron hincapié en el registro de los motivos representados para la definición de estilos y la construcción de una cronología del arte a partir de los mismos (Menghin 1952, 1957; Cardich 1979; Casamiquela 1981; Gradin \& Aguerre 1984; Gradin 1985), mientras que las últimas han focalizado la investigación integrando la información arqueológica disponible para los sitios considerados, principalmente desde una perspectiva que contempla al paisaje como un constructo social (Miotti et al. 1999, 2006, 2007; Miotti 2006; Carden 2008a, 2008b; Carden et al. 2009).

Este trabajo tiene como referente arqueológico de estudio los petroglifos de la localidad Piedra Museo, ubicada en la meseta Central de Santa Cruz. Si bien los motivos y las técnicas de los sitios que la integran han sido estudiados en anteriores oportunidades y abordados desde diferentes perspectivas (Miotti 1991;

* Rocío V. Blanco, División de Arqueología, Museo de Ciencias Naturales, La Plata-Universidad Nacional de La Plata. Paseo del Bosque s/n CP B1900, La Plata, Argentina, email: rovablanco@gmail.com

** Virginia Lynch, División de Arqueología, Museo de Ciencias Naturales, La Plata-ANPCyT. Paseo del Bosque s/n CP B1900, La Plata, Argentina, email: lynchvirginia@gmail.com 
Miotti \& Carden 2007; Carden 2008a, 2008b), aquí son considerados desde el punto de vista de la tecnología. Es decir, desde los procesos de producción de ese arte, para lo cual se utilizó el método experimental de replicación de imágenes arqueológicas como modo de establecer enunciados puente que tengan implicancia interpretativa entre las acciones del pasado y lo observado de las mismas en el presente. La localidad está compuesta por tres sitios arqueológicos (Miotti 1991; Miotti \& Salemme 1999; Carden 2008a, 2008b, 2009). De estos, solo en dos se registró arte rupestre: Alero El Galpón 2 y la Cueva Grande, ambos con pinturas y grabados (serán denominados AEG-2 y CG de ahora en adelante). En AEG-2 se registraron pinturas en el techo y las paredes del alero, así como un bloque grabado que tiene 2,3 $\mathrm{m}$ de largo por 1,2 $\mathrm{m}$ de ancho, con motivos grabados, el 70\% de los mismos son figurativos (Carden 2008a), mientras que los motivos de CG también están al interior del alero homónimo sobre 13 bloques desprendidos del techo con dimensiones variables, de los cuales siete son arqueológicos. En ambos casos, para la realización de los motivos se aprovecharon las superficies horizontales disponibles, a una altura no superior a $1 \mathrm{~m}$ (Carden 2008a).

Si bien AEG-2 y CG son los únicos sitios que presentan manifestaciones rupestres dentro de la localidad Piedra Museo, el mayor cúmulo de materiales arqueológicos ha sido recuperado de un tercer sitio: Alero El Puesto 1 (AEP-1), contiguo a AEG-2. Las excavaciones llevadas a cabo en este último fueron realizadas mediante la identificación de capas naturales, habiéndose diferenciado seis unidades depositacionales (Zárate et al. 2000; Miotti \& Salemme 2005, entre otros). De tales unidades, se han recuperado materiales líticos, arqueofaunísticos y sustancias colorantes que han sido tratados oportunamente (Cattáneo 2002, 2004; Miotti \& Salemme 2005; Carden 2008a; Hermo 2008; Miotti 2008). Entre los escasos instrumentos recuperados ninguno se ha relacionado directamente con la elaboración de los grabados rupestres (Cattáneo 2002; Carden 2008a; Lynch et al. 2010).

Es de acuerdo a lo planteado anteriormente que esta investigación tiene dos objetivos principales: en primer lugar, la caracterización de las técnicas empleadas en la localidad a través de la replicación de marcas grabadas sobre el soporte rocoso, y en segundo término, la determinación de los distintos tipos de huellas de uso dejadas en los instrumentos de sílice utilizados en tales experimentos. De este manera, se intenta profundizar el análisis de los procesos de producción y uso del arte rupestre y su vinculación con la apropiación y transformación social y simbólica del paisaje por parte de sociedades cazadoras-recolectoras que habitaron estas regiones, tema que ha sido intensamente abordado dentro del equipo de trabajo (Carden 2008a; Hermo 2008; Miotti 2008).

\section{ANTECEDENTES}

La localidad Piedra Museo se halla ubicada en la meseta Central de Santa Cruz y corresponde, en parte, al afloramiento relicto de una ingresión marina producida durante el Período Terciario (Eoceno) (ver fig. 1). Dicha ingresión se conoce como formación Puesto del Museo (Panza 2001), y está conformada por sedimentitas integradas por areniscas coquinoides. En su viaje a la localidad, Aparicio (1935: 83) la describió como una

[...] roca detrítica, porosa, friable, de color pardo grisáceo, con partes más compactadas, de color gris parduzco oscuro; constituida por pequeñas escamas finas de detrito conchil [...] dispuesto en capitas entrecruzadas y mezclado con poca arena de sílice y glauconia y muy escasos materiales arcilloides ferríferos de color ocre obscuro [...]

Además, haciendo mención a las técnicas empleadas para la realización de los motivos Aparicio (1935: 85) plantea que las líneas se pueden haber realizado "mediante una sucesión de puntos que luego se unieron entre sí. Las líneas punteadas que aparecen no son, posiblemente, sino líneas en un primer estadio de grabación". Este fue el único registro publicado hasta que, a finales de 1980, Miotti comenzó las investigaciones sistemáticas en la localidad, las cuales continuaron durante la década de 1990 (Miotti 1991, 1996; Miotti \& Cattáneo 1997; Miotti \& Salemme 1999, 2005; Cattáneo 2002; Miotti et al. 2009).

Como resultado de su composición mineralógica, la roca de la Formación Puesto del Museo es bastante porosa y friable (escala Mohs 3-4). A este factor se debe que los grabados de Piedra Museo presenten surcos profundos (entre 1 y $3 \mathrm{~cm}$ ) en comparación con los trazos más superficiales de los motivos realizados sobre bardas basálticas registrados en el área (Miotti 1991; Carden 2008a, 2008b). Carden (2008a) infiere las técnicas empleadas en la producción de grabados a partir de la observación macroscópica de los surcos. Dichas técnicas incluyen el picado, la horadación y la incisión, aunque estas tres también pueden aparecer combinadas en un mismo motivo (Carden 2008a: 319 y ss.). Además menciona que existe la posibilidad de que también se haya utilizado la "abrasión" y el machacado. ${ }^{1}$

La experimentación constituye una herramienta de gran importancia heurística para la construcción de inferencias sobre actores sociales, agentes y procesos 

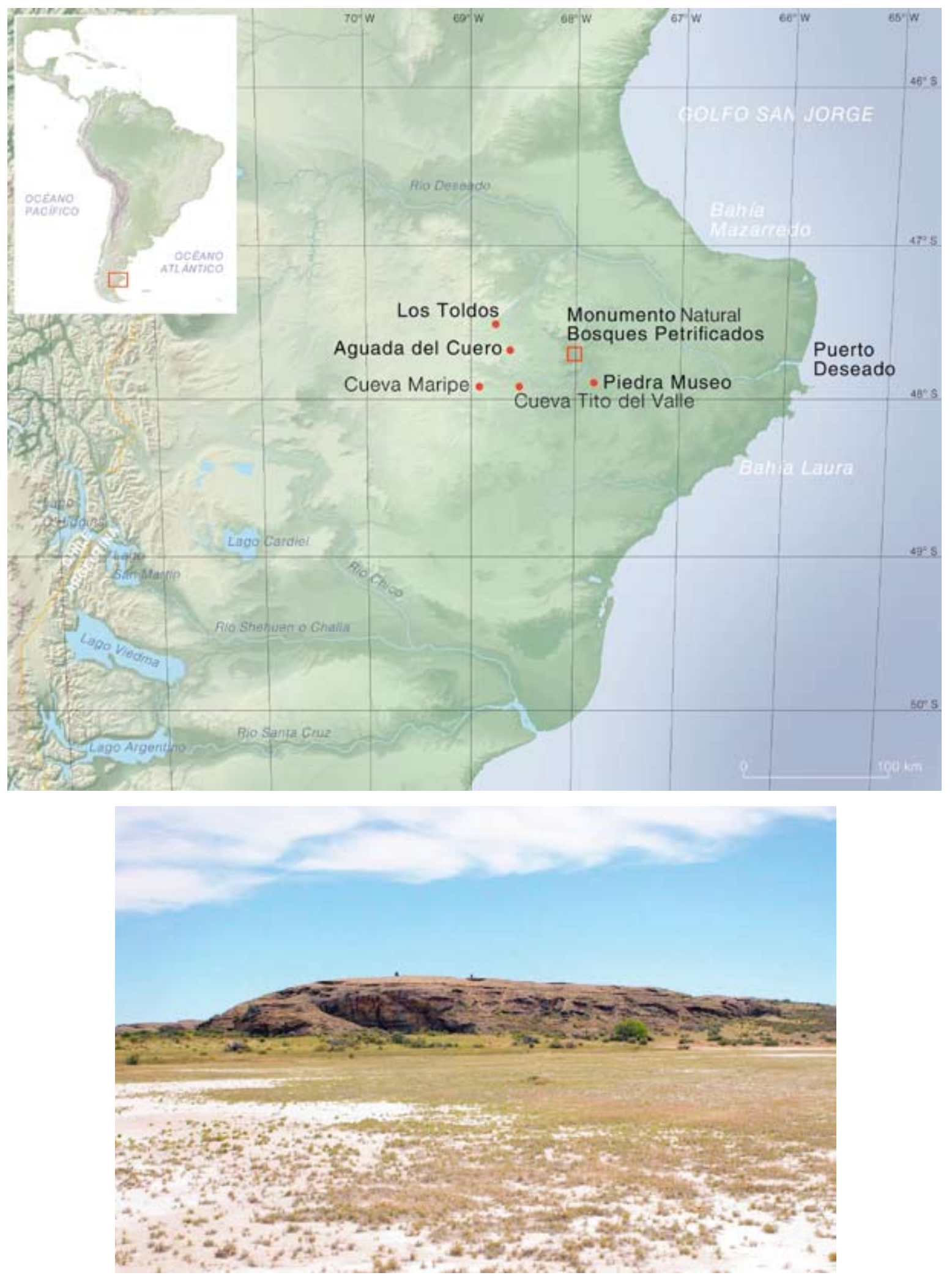

Figura 1. Piedra Museo en la meseta Central de Santa Cruz.

Figure 1. Piedra Museo (Museum Rock) on the central plateau of Santa Cruz. 
naturales del pasado. Dentro de la actividad arqueológica permite la elaboración, el desarrollo y la contrastación de hipótesis así, como el manejo controlado de variables. Es así que, dentro de la disciplina, este método ha permitido la resolución de problemáticas relacionadas a comportamientos culturales (Ascher 1961; Bordes \& Crabtree 1969) y a fenómenos naturales, que permiten la delimitación de nuevos atributos observables, o bien la redefinición de los ya conocidos, en diferentes materiales (Álvarez \& Fiore 1995).

A nivel internacional, alrededor de la década de 1960 la experimentación fue utilizada con mayor frecuencia y rigor epistemológico en diferentes campos de la disciplina (Trigger 1992). En Argentina, esta metodología fue utilizada desde los comienzos del siglo xx, para ser referida a la producción de artefactos líticos (Ameghino 1915). Con posterioridad, sufrió un importante abandono y solo fue retomada con mayor énfasis a comienzos de la década de 1980. Los estudios sistemáticos en el campo experimental han sido desarrollados principalmente en aspectos zooarqueológicos (Miotti 1992, 1994 Ms, 1995, 1998), tafonómicos (Borrero 2005) y en diferentes investigaciones acerca de la tecnología lítica (MansurFranchomme 1983, 1987; Nami 1991, 2007; Castro de Aguilar 1993). Se remarca que esta estrategia no ha sido aplicada reiteradamente al análisis del arte rupestre en Argentina (ver Álvarez \& Fiore 1995; Fiore \& Hernández Llosas 2007). Los trabajos más destacables referidos a la replicación de los motivos pintados son los efectuados por Paunero $(1992,1994)$. En cuanto a los casos aplicados al estudio de las técnicas en la producción de grabados rupestres, se cuenta con los trabajos de Álvarez y Fiore (1995), Álvarez y colaboradores (1999, 2001), Fiore (1996-1998, 1999, 2007). Álvarez y Fiore (1995) realizan una replicación de motivos rupestres grabados a partir de un diseño experimental y sugieren la posibilidad de su realización mediante artefactos no formatizados, sobre todo teniendo en cuenta la escasez de cinceles en el registro arqueológico. Por otro lado, a partir de la realización de experimentos preliminares, Fiore (2007) plantea que las técnicas empleadas para la producción de motivos grabados están muy relacionadas a la dureza del soporte, sea para minimizar el tiempo o la inversión de energía en el trabajo a realizar o bien para reducir el daño de los artefactos utilizados.

Dentro del análisis funcional, en Argentina y desde la década de 1980, se han llevado a cabo importantes aportes al conocimiento de este aspecto de la disciplina arqueológica (Mansur-Franchomme 1983, 1987; Castro de Aguilar 1993; Álvarez et al. 1999, 2001; Álvarez 2003; Leipus 2006). En lo referente a esta vía analítica, y vinculado al estudio de artefactos empleados para la producción de imágenes rupestres, solo podemos mencionar los trabajos realizados por Álvarez y colaboradores (1999, 2001), quienes caracterizaron los rastros de uso en artefactos de basalto y obsidiana resultantes del trabajo sobre soporte tobáceo, mediante la observación de dichas piezas experimentales a partir de un microscopio metalográfico y MEB (microscopio electrónico de barrido).

En este sentido, y en función de los antecedentes mencionados, el trabajo aquí presentado constituye un importante aporte a los estudios experimentales acerca de la producción de marcas grabadas, puesto que incorpora nuevos datos tanto con relación a la variabilidad de soportes trabajados como también con relación a la materia prima utilizada para la confección de los instrumentos.

\section{MATERIALES Y MÉTODOS}

La realización del trabajo experimental consistió en la utilización de tres técnicas que fueron definidas inicialmente por Álvarez y Fiore (1995: 218-219):

Incisión: implica el corte o hendidura del soporte mediante el movimiento unidireccional del artefacto, cuyo filo se desplaza en forma paralela a la dirección de la utilización.

Raspado: se trata de la abrasión del soporte mediante el movimiento bidireccional del artefacto, cuyo filo se desplaza en forma perpendicular a la dirección de utilización. ${ }^{2}$

Horadación: implica la inserción gradual de un artefacto en el soporte mediante movimientos de rotación en sentido horario $\mathrm{y}$ antihorario.

Según dichas autoras, estas tres técnicas se definen por el tipo de mecánica ejercida, en función del movimiento y del artefacto necesario para realizarla, por lo que las agrupan en aquellas que requieren presión. Si bien Carden (2008a) infiere que las técnicas usadas con mayor frecuencia para la realización de motivos grabados en Piedra Museo son el picado y el machacado, en esta oportunidad tales técnicas no han sido contempladas. Esto último se debe a que la aplicación de las mismas produce fracturas en los bloques experimentales utilizados para la experimentación en laboratorio, consecuencia de su escasa dureza y grosor.

Las experiencias replicativas fueron llevadas a cabo en el laboratorio, sobre tres bloques pertenecientes a la Formación Puesto del Museo, recolectados en campo en diferentes campañas arqueológicas (fig. 2). Como resultado de su composición mineralógica, la consistencia de esta coquina y su baja dureza ofrecieron poca resistencia ante la presión para realizar marcas.

Dado que la mayoría de las representaciones grabadas se registran sobre la cara horizontal de los bloques, se 

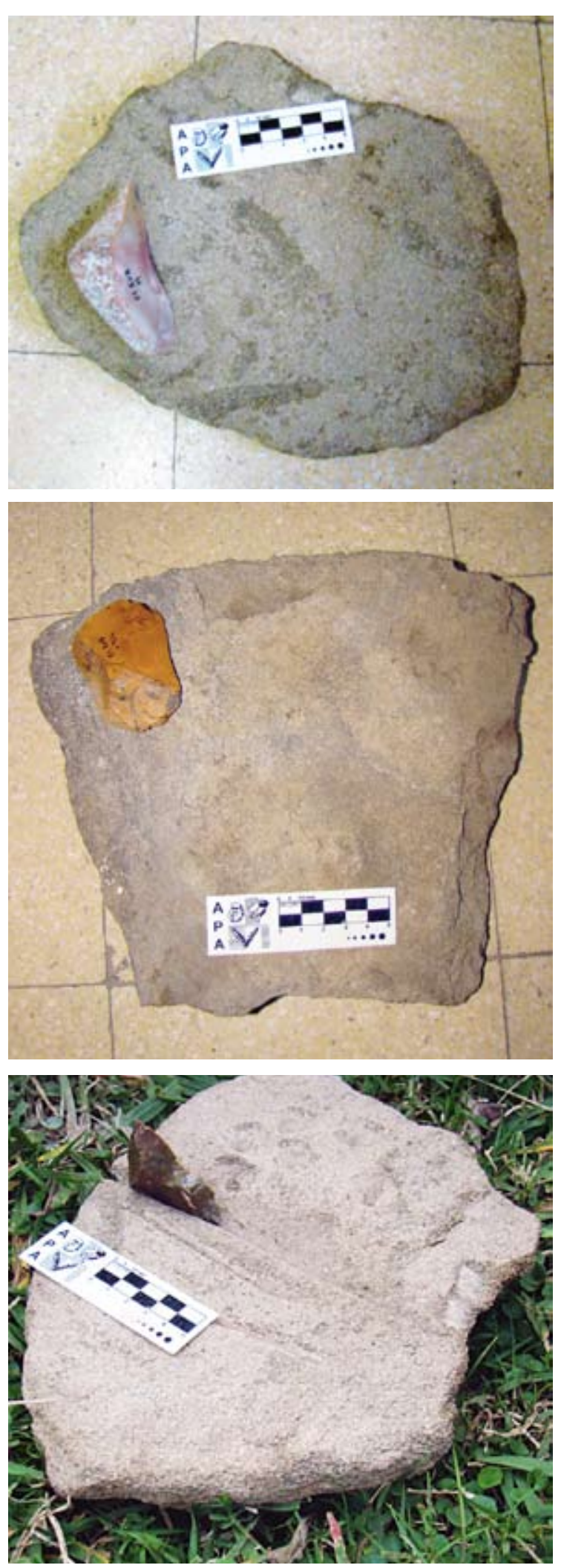

Figura 2. Bloques experimentales previa utilización. Figure 2. Experimental blocks before carving. decidió practicar las marcas respetando dicha posición en los bloques experimentales. El aspecto que varió fue la altura con respecto al suelo, ubicándolos al ras del mismo en el transcurso del trabajo.

Las marcas fueron realizadas por dos mujeres diestras, sentadas en el suelo con un campo manual hacia el interior (sensu Álvarez \& Fiore 1995). La prensión de los artefactos fue directa, sin enmangue (figs. 3 y 4 ). En total se efectuaron 42 marcas, utilizando las técnicas mencionadas anteriormente. De ese total, seis marcas corresponden a la técnica de incisión, nueve, al raspado y 27 , a la técnica de horadación. Debemos señalar que esta variación en el número de marcas realizadas por técnica se relaciona con los períodos temporales de trabajo propuestos que se detallan más adelante. La morfología de los surcos obtenidos en el transcurso de las experiencias es en "V" para incisión y en "U" para raspado y horadación, coincidente con la ya informada por Álvarez y Fiore (1995 y bibliografía allí citada).

Por otro lado, cabe aclarar que para aprovechar mejor la superficie de los bloques, las marcas fueron realizadas sobre ambas caras disponibles, siendo denominadas "Cara A" y "Cara B", respectivamente.

Las piezas experimentales empleadas en esta oportunidad corresponden a artefactos no formatizados (ANF, Aschero \& Hocsman 2004) confeccionados en sílice por percusión directa, utilizando un percutor duro (fig. 5). La selección de esta materia prima se debe a que el registro arqueológico de la localidad Piedra Museo está compuesto en su mayor parte por artefactos silíceos, además de que la misma se encuentra disponible en el área. La colección fue medida utilizando los criterios pautados por el equipo de trabajo (Hermo 2008), dibujada, fotografiada y observada bajo lupa binocular y microscopio metalográfico, con anterioridad a su utilización. El número de piezas experimentales fue de 15 lascas de filo natural, que fueron utilizadas por períodos de cinco, 15, 30, 45 y 60 minutos para cada una de las técnicas antes descritas. Dichos lapsos temporales fueron no aditivos; es decir, para cada técnica contamos con ANF que fueron trabajados únicamente durante los períodos detallados (Tabla 1). El tamaño de las piezas empleadas fue de grande a muy grande (Aschero 1975 Ms), con un filo potencialmente utilizable mayor a $30 \mathrm{~mm}$. La delineación así como el ángulo de los filos se detallan en la Tabla 1.

La posición del filo con respecto a la superficie, la dirección del movimiento y el ángulo de trabajo están determinados por la técnica empleada. En el caso de la incisión, la posición del filo es paralela respecto al movimiento del instrumento sobre la superficie trabajada, la dirección del movimiento es unidireccional interior y 
Tabla 1. Detalle de las experiencias.

Table 1. Detail of the experiments.

\begin{tabular}{|c|c|c|c|c|c|c|c|c|c|}
\hline Técnica & Tiempo & $\begin{array}{l}\text { Bloque } \\
\text { experimental }\end{array}$ & Lado & ANF* & $\begin{array}{l}\text { Delineación } \\
\text { del filo }\end{array}$ & $\begin{array}{l}\text { Ángulo } \\
\text { del filo }\end{array}$ & Ejecutante & Prensión & $\begin{array}{c}\text { Cantidad } \\
\text { de marcas }\end{array}$ \\
\hline \multirow{5}{*}{ Incisión } & 5 & \multirow{3}{*}{ BE 1} & \multirow{3}{*}{ Cara A } & E1 RVB 14 & Convexo & 35 & Mujer, diestra & Directa & 1 \\
\hline & 15 & & & E1 LM 13 & Convexo & 15 & Mujer, diestra & Directa & 2 \\
\hline & 30 & & & E1 RVB 44 & Rectilíneo & 50 & Mujer, diestra & Directa & 1 \\
\hline & 45 & \multirow{2}{*}{ BE 2} & \multirow{2}{*}{ Cara A } & E1 RVB 42 & Rectilíneo & 60 & Mujer, diestra & Directa & 1 \\
\hline & 60 & & & E1 RVB 48 & Convexo & 30 & Mujer, diestra & Directa & 1 \\
\hline \multirow{5}{*}{ Raspado } & 5 & BE 2 & Cara B & E1 RVB 24 & Convexo & 15 & Mujer, diestra & Directa & 1 \\
\hline & 15 & BE 3 & Cara B & E1 RVB 29 & Convexo & 25 & Mujer, diestra & Directa & 6 \\
\hline & 30 & BE 2 & Cara B & E1 LM 17 & Convexo & 25 & Mujer, diestra & Directa & 1 \\
\hline & 45 & \multirow{2}{*}{ BE 1} & \multirow{2}{*}{ Cara B } & E1 RVB 43 & Rectilíneo & 50 & Mujer, diestra & Directa & \multirow{2}{*}{1} \\
\hline & 60 & & & E1 RVB 41 & Rectilíneo & 35 & Mujer, diestra & Directa & \\
\hline \multirow{5}{*}{ Horadación } & 5 & BE 2 & Cara B & E1 RVB 60 & - & - & Mujer, diestra & Directa & 1 \\
\hline & 15 & \multirow{2}{*}{ BE 3} & \multirow{2}{*}{ Cara A } & E1 RVB 11 & - & - & Mujer, diestra & Directa & 9 \\
\hline & 30 & & & E1 RVB 17 & - & - & Mujer, diestra & Directa & 9 \\
\hline & 45 & BE 1 & Cara A & E1 RVB 45 & - & - & Mujer, diestra & Directa & 6 \\
\hline & 60 & BE 2 & Cara A & E1 RVB 47 & - & - & Mujer, diestra & Directa & 2 \\
\hline
\end{tabular}

Referencias: BE: Bloque experimental. ANF: Artefacto no formatizado.

* El siglado de los ANF respeta la numeración correspondiente a la colección experimental, por lo tanto no es continuo.

el ángulo de trabajo es de $90^{\circ}$. En el raspado, la posición del filo es perpendicular respecto al movimiento del instrumento sobre la superficie trabajada, el movimiento es bidireccional y el ángulo de trabajo es de 90. La horadación merece una mención especial, ya que si bien la posición del artefacto es perpendicular a la superficie trabajada, el movimiento de rotación genera que entren en contacto el vértice y los filos laterales del artefacto. Con respecto al ángulo de trabajo la posición inicial del vértice es de $90^{\circ}$, pero a medida que se va profundizando la marca dicho ángulo varía.

\section{Análisis funcional de la serie experimental}

Para el estudio de los microrrastros, de los 15 ANF se observaron 25 filos de ambas caras (dorsal y ventral) siendo utilizado para los casos de incisión y raspado un solo filo por artefacto; mientras que en el caso de la horadación intervinieron el vértice y de dos a tres filos por pieza. Luego de cada experimento, las piezas fueron lavadas con agua jabonosa y alcohol con el fin de remover los residuos presentes sobre las superficies y permitir una mejor visibilidad bajo el microscopio.
El instrumental lítico fue examinado bajo un microscopio metalográfico Nikon Epiphoto 200, bajo el campo de iluminación claro con objetivos y oculares que permiten una magnificación de 50 hasta 500x. Mientras que para la observación de esquirlamientos se utilizó una lupa binocular Nikon SMZ 800 con una magnitud de 10 hasta 63x. Las variables dependientes contempladas fueron los micropulidos, las estrías, los esquirlamientos y el redondeamiento del filo (Semenov 1964; Keeley 1980; Anderson-Gerfaud 1981; Vaughan 1985).

Los micropulidos fueron definidos por Keeley en 1977 y considerados como aspectos de la superficie de un filo utilizado, que refleja la luz incidente de un modo distinto a las zonas no trabajadas; permiten determinar el tipo de material sobre el cual un instrumento ha sido usado. Los esquirlamientos, en tanto, son modificaciones de los filos como consecuencia de fracturas, que alteran la sección longitudinal o delineación de los mismos (Keeley 1980). No están necesariamente asociados al uso de un artefacto. Por último, las estrías son consideradas rastros lineares que ocurren sobre la superficie de un instrumento al ser utilizado, permitiendo, en conjunto con la observación del micropulido, determinar la 

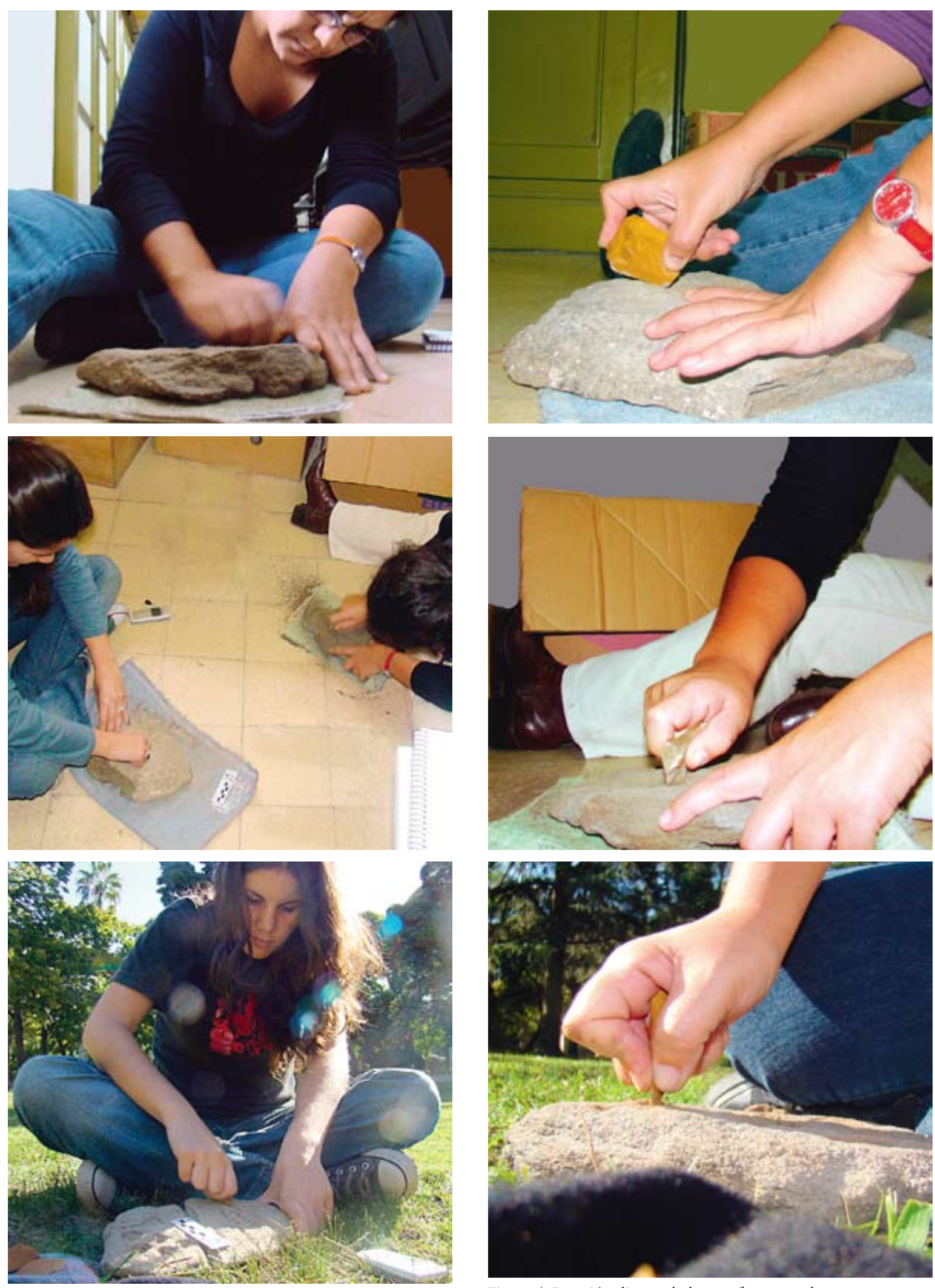

Figura 3. Posición de las ejecutantes y ámbito de las experiencias Figure 3. Carvers' positioning and context.

Figura 4. Prensión directa de los artefactos usados. Figure 4. Applying direct pressure with stone artifacts. 

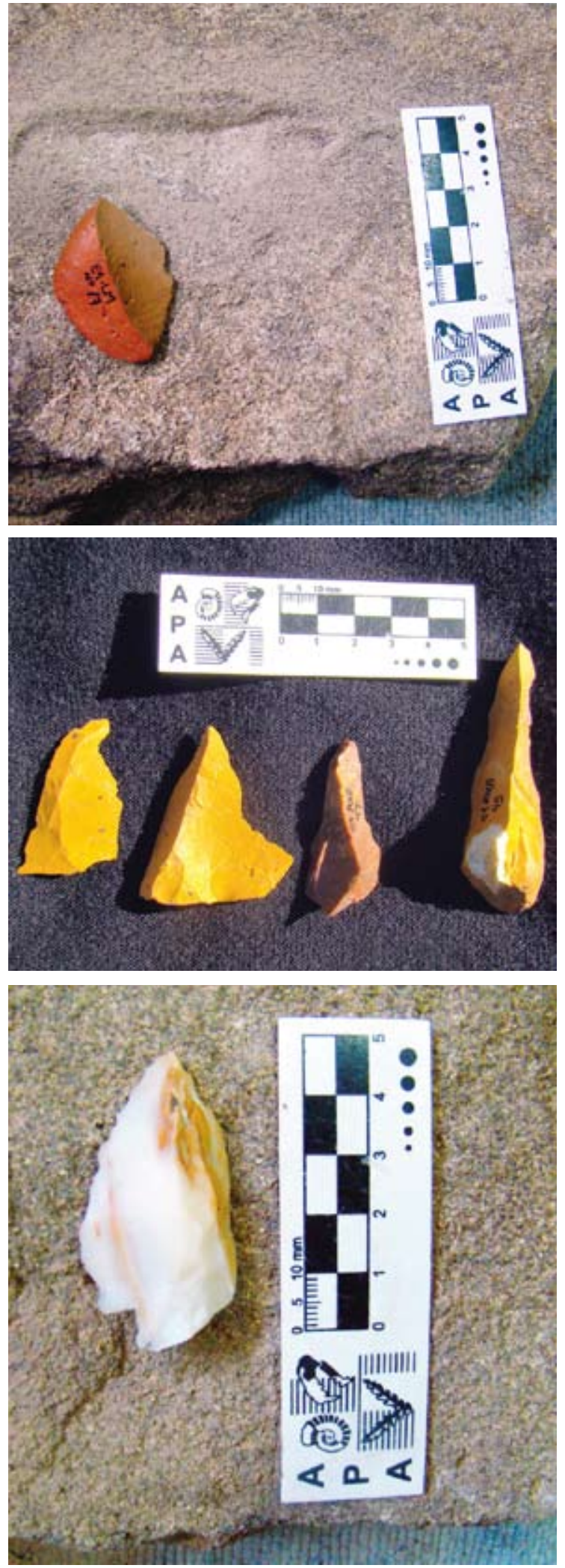

Figura 5. Artefactos empleados durante las experiencias. Figure 5. Artifacts used for the replication experiments. direccionalidad del trabajo efectuado. La formación de estrías está condicionada por varios factores como son la presencia de abrasivos y los desprendimientos de microlascas durante el empleo de un artefacto (MansurFranchomme 1983, 1987; Álvarez 2003). En la Tabla 2 se detallan las variables consideradas para cada rastro mencionado anteriormente.

\section{RESULTADOS}

Siguiendo la lógica experimental podría enunciarse que los motivos arqueológicos pueden haber sido ejecutados utilizando ANF, los cuales son de fácil prensión sin necesidad de enmangue para la realización del trabajo. El filo potencialmente utilizable mayor a $30 \mathrm{~mm}$ en dichos artefactos fue de gran ayuda para dejar marcas sobre el soporte, facilitando su manejo y aumentando la superficie de contacto con aquel, lo cual ayudó en la posterior detección de los rastros de uso.

Con relación a los resultados obtenidos a partir del análisis de los rastros de uso de las piezas experimentales, pudimos determinar desde los cinco minutos de comenzado el trabajo las etapas iniciales de modificación de las superficies de los filos.

En todas las piezas empleadas para las técnicas de incisión y raspado se produjo contacto con el material trabajado en ambas caras del filo, por lo que el desarrollo del micropulido implicó tanto la cara dorsal como la ventral.

A los cinco minutos, todas las piezas tenían un buen desarrollo de micropulido sobre ambas caras, lo que en conjunto con la orientación del mismo (paralela al filo trabajado), permitió identificar la cinemática efectuada. En la incisión las características generales que presentó el micropulido fueron las siguientes: brillo muy brillante, regularidad intermedia, espesor intermedio a espeso, desarrollo del micropulido sobre las superficies altas y bajas de la microtopografía del filo y extensión intermedia. En el caso de esquirlamientos podemos decir que en los primeros minutos de la actividad se observó una mayor cantidad de los mismos sobre el filo, una vez estabilizado el movimiento pasaron a ser medianos a escasos y discontinuos, probablemente como consecuencia de la escasa dureza del soporte trabajado. En su mayoría presentaron una morfología trapezoidal con terminaciones escalonadas. En todas las piezas utilizadas para la incisión se determinó la presencia de estrías con morfología variable y una orientación paralela al filo utilizado, permitiendo así una mejor identificación del movimiento realizado. Al igual que en la técnica de raspado, se observó redondeamiento del filo en 
Tabla 2. Variables contempladas en el análisis de rastros de uso.

Table 2. Variables taken into account in the microwear analysis.

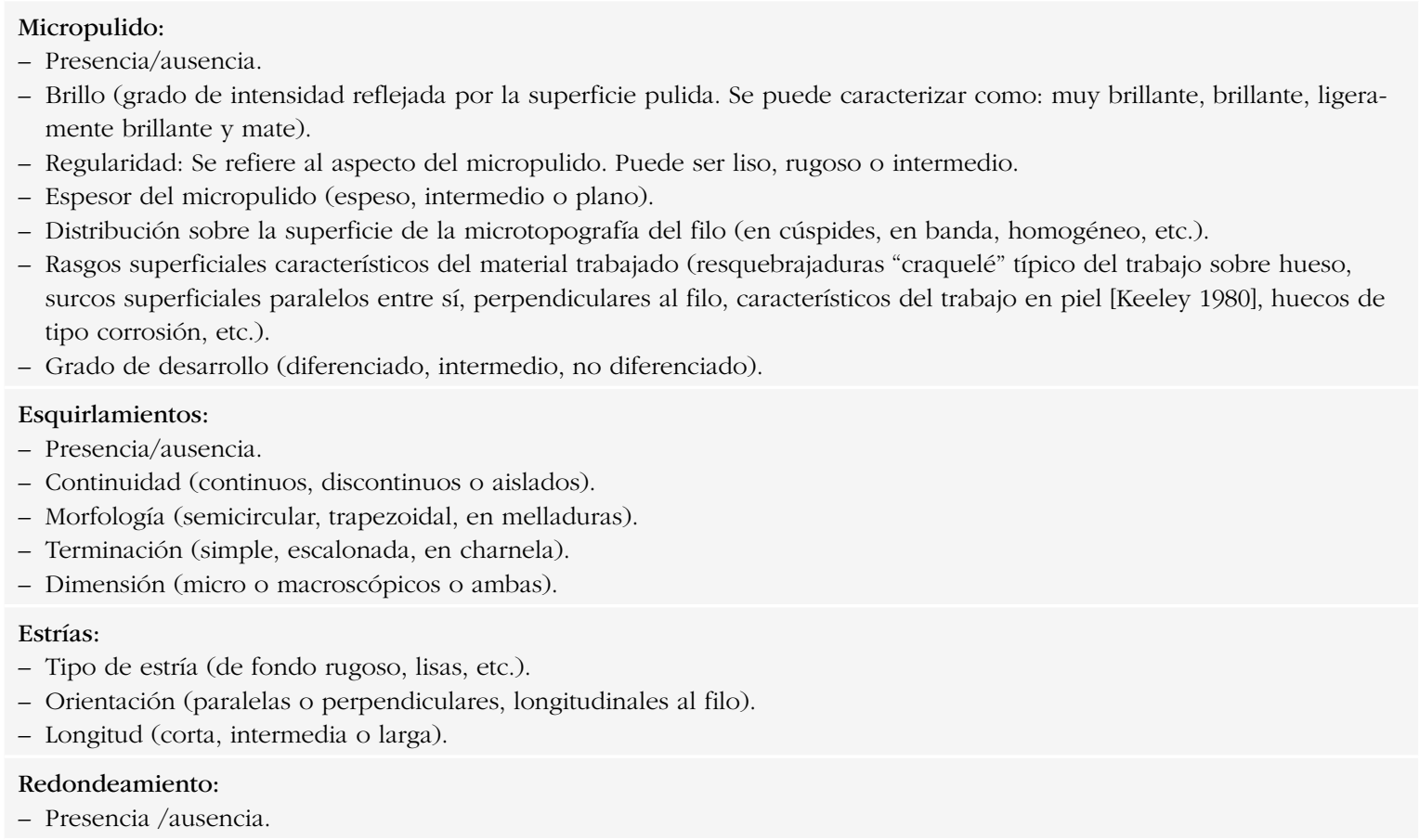

aquellas piezas que estuvieron trabajadas a partir de 15 minutos (fig. 6).

Para la técnica de raspado se observó micropulido con características diagnósticas a partir de 15 minutos de realizada la actividad. El mismo presentó brillo muy brillante, regularidad intermedia, orientación transversal al filo, espesor intermedio a espeso, una distribución en las superficies altas en los grupos de trabajo de 15 y 30 minutos y homogéneo en los grupos de 45 y 60 minutos, consecuencia de las etapas iniciales características del desarrollo del micropulido. La presencia de esquirlamientos fue de abundante a escasa, discontinua y de morfología trapezoidal con terminaciones escalonadas. Se pudo identificar la presencia de estrías, las cuales fueron de morfología variable y con una direccionalidad perpendicular al filo utilizado (fig. 7).

A partir de la distribución de las estrías y el micropulido desarrollado en los filos que intervinieron durante la actividad efectuada, en la técnica de horadación se determinaron dos tipos de movimientos. Por un lado, un movimiento transversal o transversal-oblicuo, en especial en la porción medial del filo, donde se detectaron estrías de longitud y morfología variable con una orientación transversal-oblicua al filo. Por otro lado, se observaron movimientos rotatorios en la arista distal del instrumento (punto de apoyo).
A los cinco minutos de iniciada la actividad, los cristales presentes habían sufrido modificaciones debido al contacto con el material mineral. Aun así -salvo en la sección del vértice, donde se identificaron los estadios iniciales del micropulido-, en los demás filos que intervinieron no pudo determinarse la formación de estrías ni micropulidos, pero sí de abundantes esquirlamientos (continuos, semicirculares-trapezoidales y de terminación escalonada).

A partir de los 15 minutos hubo buen desarrollo de micropulido, con un brillo mate a ligeramente brillante, orientación transversal-oblicua, espesor intermedio y una distribución en las superficies altas de la microtopografía del filo. Los esquirlamientos registrados variaron de medianos a escasos, discontinuos, de morfología trapezoidal o semicircular con terminaciones escalonadas y simples. En esta actividad también se observó un mayor número de piezas con redondeamiento del filo, en comparación con aquellas utilizadas tanto para la incisión como para la técnica de raspado (fig. 8).

\section{CONCLUSIONES}

Dado que en el conjunto lítico de estratigrafía del sitio Alero El Puesto 1 no aparecen artefactos inferidos 

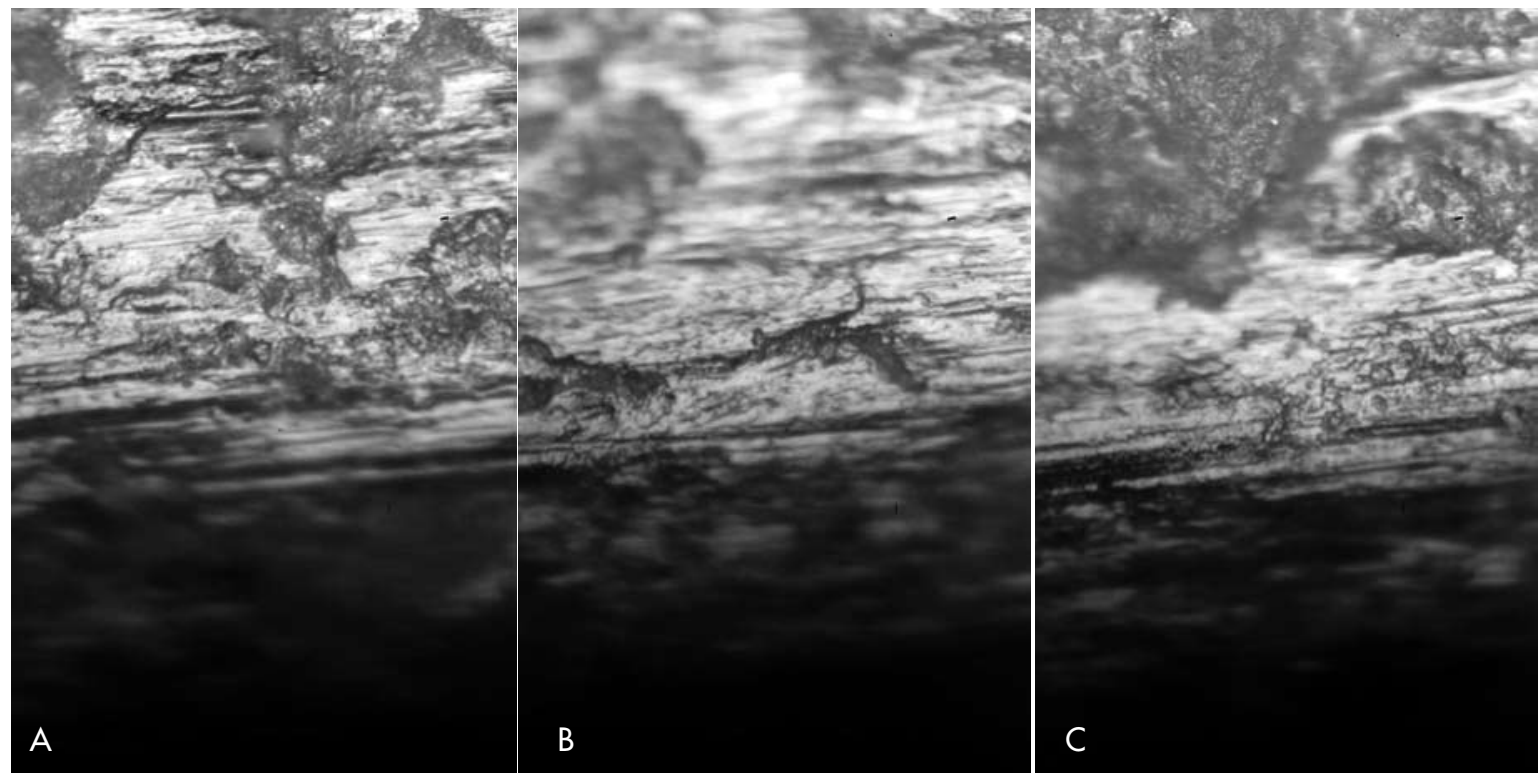

Figura 6. Micropulido resultante de la técnica de incisión: a) Pieza trabajada 45' con movimiento bidireccional. Magnificación: 200x. Campo de iluminación claro; b) Pieza trabajada 60' con movimiento bidireccional. Presencia de estrías de morfología variable. Magnificación: 200x. Campo de iluminación claro; c) Pieza trabajada 60' con movimiento bidireccional. Magnificación: 200x. Campo de iluminación claro.

Figure 6. Micropolish resulting from incision technique: a) Piece worked for 45' with bidirectional action. Magnification: $200 x$. Bright field illumination; b) Piece worked for 60' with bidirectional action. Presence of grooves with variable morphology. Magnification: 200x. Bright field illumination; c) Piece worked for 60' with bidirectional action. Magnification: 200x. Bright field illumination.
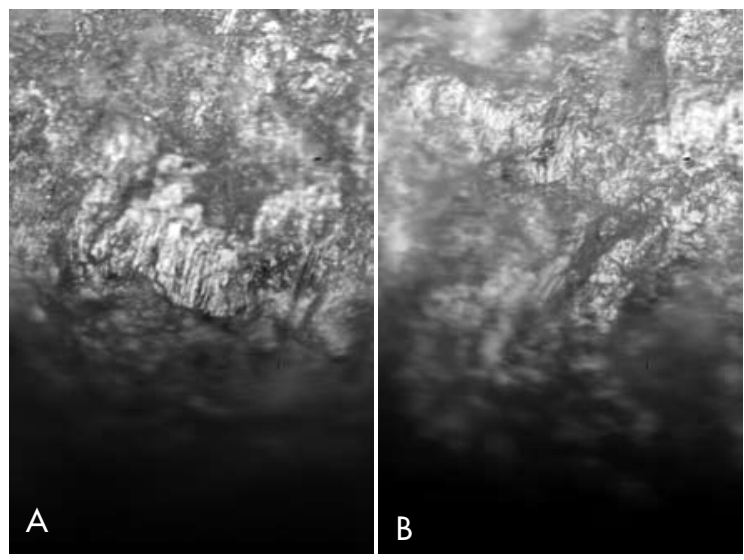

Figura 7. Micropulido resultante de la técnica de raspado sobre soporte rocoso de arenisca coquinoide: a) Pieza trabajada 60 con movimiento bidireccional. Magnificación: 200x. Campo de iluminación claro; b) Pieza trabajada 45'. La presencia de estrías permite identificar el movimiento realizado transversalmente al filo. Magnificación: 200x. Campo de iluminación claro.

Figure 7. Micropolish resulting from scraping technique on coquinoid sandstone: a) Piece worked for 60' with bidirectional action. Magnification: 200x. Bright field illumination; b) Piece worked for 45'. The presence of grooves shows the direction of movement across the edge. Magnification: 200x. Bright field illumination para realizar grabados, p. e., percutor/cincel (Cattáneo 2002), es altamente probable que las representaciones hayan sido elaboradas con ANF. Es a partir de este supuesto que se consideró necesario emplear estos artefactos en el desarrollo del programa experimental. De acuerdo a estas consideraciones, y contemplando la hipótesis propuesta por Álvarez y Fiore (1995), es posible la producción de motivos grabados a partir de un solo instrumento, el cual no necesariamente tiene que ser sofisticado o diseñado especialmente para tal efecto. En tal sentido, aumenta nuestra expectativa respecto a la probabilidad de que los ANF hayan sido utilizados para estos fines. Así, hallar en el registro arqueológico artefactos específicos para la consecución de imágenes grabadas es por ahora menos probable y justifica en parte su ausencia. Los materiales arqueológicos de Piedra Museo están actualmente en vías de estudio mediante la metodología del análisis funcional, por lo que la experimentación llevada a cabo en el presente trabajo es una herramienta fundamental a la hora de identificar posibles rastros de uso sobre material mineral.

Por otro lado, las experiencias efectuadas han permitido realizar una primera caracterización de huellas de uso que resultan de la implementación de las técnicas 

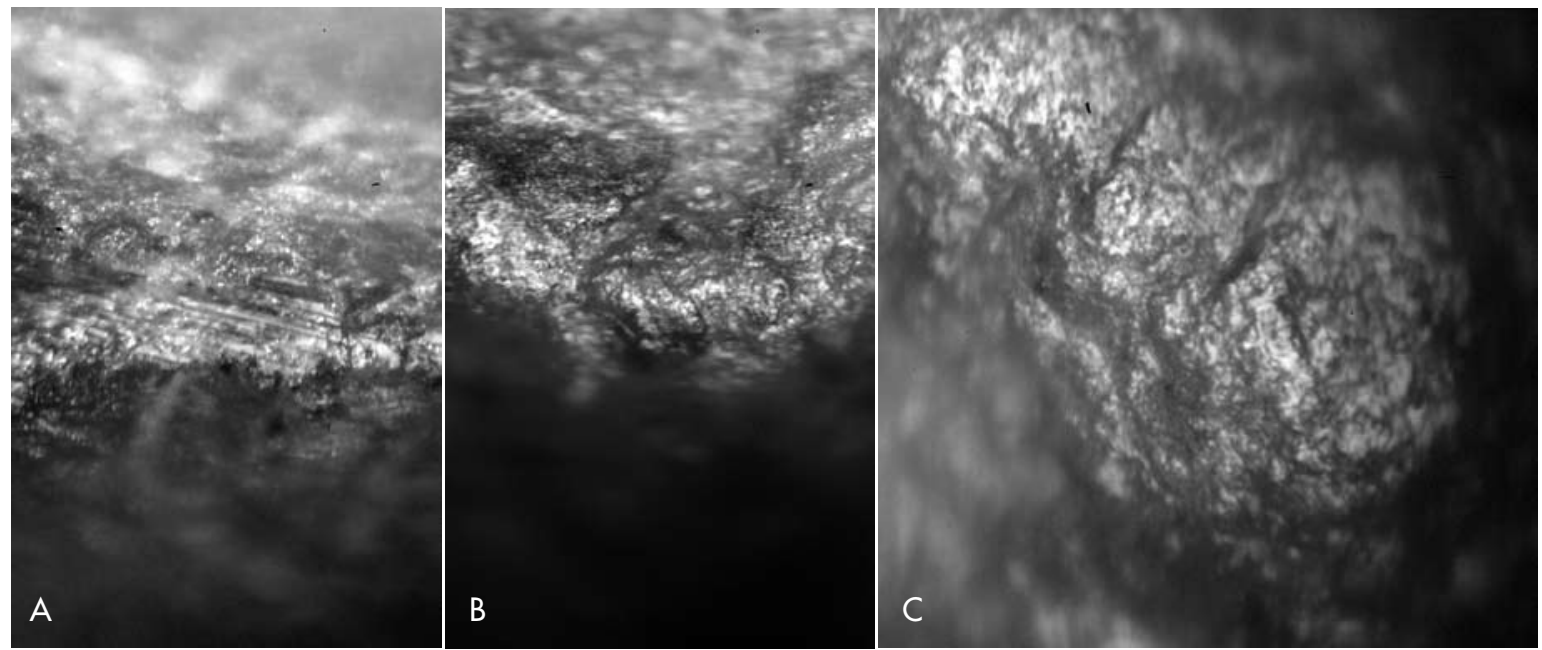

Figura 8. Rastros microscópicos resultantes de la técnica de horadación: a) Se puede observar micropulido en los filos laterales de la pieza trabajada 45' en la técnica de horadación. Las estrías nos permiten inferir el movimiento realizado (transversal oblicuo). Magnificación: 200x. Campo de iluminación claro; b) Pieza trabajada 60' en horadación. Se puede distinguir un fuerte redondeamiento en la cara ventral del punto de apoyo de la pieza. Magnificación: 200x. Campo de iluminación claro; c) Pieza trabajada 60' en horadación. Fuerte redondeamiento del filo en el punto de apoyo de la pieza, consecuencia de una actividad rotatoria. Magnificación: 200x. Campo de iluminación claro.

Figure 8. Microscopic traces of drilling action: a) Micropolish can be observed in the lateral edges of the worked piece for $45^{\prime}$ using a drilling technique. The grooves allow us to infer the cinematic (oblique transverse). Magnification: 200x. Bright field illumination; b) Piece worked for 60' using a drilling technique. Notable rounding can be seen on the underside, where the piece was supported. Magnification: 200x. Bright field illumination; c) Piece worked for 60' using a drilling technique. There is notable rounding of the supporting edge as a result of rotating action. Magnification: 200x. Bright field illumination.

que comportan el ejercicio de presión en la confección de motivos rupestres grabados. No obstante, aún resta por caracterizar los rastros resultantes de las técnicas que implican percusión: picado y machacado (Álvarez \& Fiore 1995).

Hasta el momento, en Argentina se encuentra poco registro experimental sobre la temática aquí tratada. Los trabajos de Álvarez y colaboradores (1999, 2001) nos han permitido formular los planteamientos iniciales en el presente trabajo. Sin embargo, difieren de aquellos con relación a las técnicas empleadas, al soporte trabajado y la materia prima con la que se confeccionaron los artefactos utilizados en la experimentación; siendo en aquel caso el soporte grabado una toba perteneciente a la Formación Collón Curá y la colección experimental corresponde a artefactos hechos en basalto y obsidiana. A pesar de estas diferencias, los rastros de uso registrados en ambos casos son característicos de las técnicas empleadas (raspado e incisión, Álvarez et al. 2001).

Podemos concluir que el análisis funcional de base microscópica en conjunto con el análisis de las cadenas operativas implicadas en la producción de imágenes grabadas, son dos vías analíticas que permitirán generar un corpus de datos actualísticos para fundar nuevas hipótesis acerca del registro arqueológico de la localidad Piedra Museo. Es a raíz de esto que el futuro trabajo de investigación incluye la realización de marcas por percusión, la replicación de motivos grabados y el posterior análisis funcional de los artefactos empleados para tal fin. Además se continuará con la revisión de las colecciones arqueológicas en busca de los rastros de uso dejados sobre los artefactos coincidentes con los aquí presentados. Como dice Bednarik (2001: 50)

The technological aspects of rock art are of just as much important as is its dating, for instance, but they have been treated in a cavalier fashion so far, and most of limited information about this subject contained in the literature is either inadequate or false. There are telling similarities in the way the methodology of Semenov (1964), which should have been such a boon for archaeology, was ignored or misunderstood for many years. It is one of the major tasks of rock art science to develop this field in the years ahead.

RECONOCIMIENTOS A nuestros compañeros de laboratorio, quienes han leído críticamente este manuscrito; a las doctoras Laura Miotti, Myrian Álvarez y Natalia Carden, por el apoyo, la lectura crítica y los consejos durante nuestras primeras etapas de análisis. Especialmente a Natalia Barreto, quien aportó su trabajo para las experiencias en laboratorio. Hacemos una mención especial a los evaluadores anónimos, quienes con sus sugerencias contribuyeron a la mejora de esta publicación. Este trabajo fue financiado por la Agencia Nacional de Promoción Científica y 
Tecnológica (ANPCYT- PICT 1552) y la Universidad Nacional de La Plata (PI N/550). Todo lo escrito aquí es exclusiva responsabilidad de las autoras.

\section{NOTAS}

1 Álvarez y Fiore (1995) y Fiore (1996-1998) contemplan la abrasión como sinónimo de raspado, mientras que denominan machacado al golpeteo sin la utilización de un intermediario. Esta última técnica junto al picado se agruparían dentro de aquellas en las que se ejerce percusión para poder lograr una imagen.

${ }^{2}$ Fiore (2007) plantea que tanto la incisión como el raspado pueden ser unidireccionales o bidireccionales; lo que los diferencia netamente es la posición del filo respecto a la superficie trabajada.

\section{REFERENCIAS}

Álvarez, M., 2003. Organización tecnológica en el Canal Beagle. El caso de Túnel I (Tierra del Fuego, Argentina). Tesis doctoral de la Facultad de Filosofía y Letras, Universidad de Buenos Aires, Buenos Aires.

Álvarez, M. \& D. Fiore, 1995. Recreando imágenes: diseño de experimentación acerca de las técnicas y los artefactos para realizar grabados de arte rupestre. Cuadernos del INAPL 16: 215-239, Buenos Aires.

Álvarez, M.; D. Fiore, E. Favret \& R. Castillo Guerra, 1999. El uso de artefactos líticos para la ejecución de grabados rupestres: Observación y análisis de los rastros de utilización mediante técnicas de microscopia óptica. En Actas del XII Congreso Nacional de Arqueología Argentina, C. Diez, Ed., tomo III, pp. 327-335. La Plata: Universidad Nacional de La Plata.

- 2001. The use of lithic artifacts for making rock art engravings: observation and analysis of use-wear traces in experimental tools through optical microscopy and SEM. Journal of Archaeological Science 28: 457-464, Cambridge.

Ameghino, F., 1915. Filogenia: principios de clasificación transformista basados sobre leyes y proporciones matemáticas. Buenos Aires: La Cultura Argentina.

ANDERSON-GeRFAUd, P. C., 1981. Contribution méthodologique a l'analyse des microtraces d'utilisation sur les outils préhistoriques. Tesis doctoral de la Universidad de Bordeaux I, Bordeaux.

Aparicio, F. DE, 1935. Viaje preliminar de exploración en el territorio de Santa Cruz. Publicación del Museo Antropológico y Etnológico de la FFYL-UBA Serie A, III: 71-92, Buenos Aires.

ASCHER, R., 1961. Experimental archaeology. American Anthropologist 63: 793-816, Washington

Aschero, C., 1975 Ms. Ensayo para una clasificación morfológica de los instrumentos líticos aplicada a estudios tipológicos comparativos. Informe al Consejo Superior de Investigaciones Científicas (CONICET), Buenos Aires.

Aschero, C. \& S. Hocsman, 2004. Viejas y nuevas categorías analíticas y niveles de clasificación en el análisis macroscópico de artefactos líticos tallados. Taller Morfología macroscópica en la clasificación de artefactos líticos: Innovaciones y perspectivas [online] $<$ http://www.naya.org.ar/eventos/liticos_resumenes.htm>

BEDNARIK, R., 2001. The technology of rock art. En Rock art science. The scientific study of palaeoart, R. Bednarik, Ed., pp. 37-53. Turnhout: Brepols Publishers.

Bordes, F. \& D. Crabtree, 1969. The Corbiac blade technique and others experiments. Tebiwa 12 (2): 1-21, Idaho.

Borrero, L. A., 2005. Taphonomy of Late Pleistocene Faunas at Fuego-Patagonia. Journal of South American Earth Sciences 20 (1-2): 115-120, Columbia.

CARDEn, N., 2008a. Estudio del arte rupestre de la meseta Central de Santa Cruz. El área de los zanjones Blanco y Rojo, al sur del río
Deseado. Tesis doctoral de la Facultad de Ciencias Naturales, Universidad Nacional de La Plata, La Plata.

- 2008b. Territories among hunter-gatherers \& the ritual dimension of landscape: the Central Patagonian Plateau, Argentina. Before Farming 1: 1-19, London.

-2009. Prints on the rocks: a study of the track representations from Piedra Museo locality (Southern Patagonia). Rock Art Research 26 (1): 29-43. Melbourne: AURA.

Carden, N.; L. Magnin \& L. Miotti, 2009. Distribución de figuras animales y dinámica poblacional: un estudio comparativo en Patagonia (provincia de Santa Cruz, Argentina). En Crónicas sobre la piedra: Arte Rupestre de las Américas, M. Sepúlveda, L. Briones \& J. Chacama, Eds., pp. 153-173. Arica: Ediciones Universidad de Tarapacá.

Cardich, A., 1979. A propósito de un motivo sobresaliente en las pinturas rupestres de El Ceibo (provincia de Santa Cruz). Relaciones de la Sociedad Argentina de Antropología XIII: 163-182, Buenos Aires.

Casamiquela, R., 1981. El arte rupestre de la Patagonia. Buenos Aires: Ediciones Siringa.

Cattáneo, R. G., 2002. Una aproximación a la organización tecnología lítica entre los cazadores recolectores del Holoceno medio/Pleistoceno final en la Patagonia Austral. Tesis doctoral de la Facultad de Ciencias Naturales, Universidad Nacional de La Plata, La Plata.

— 2004. Conjuntos instrumentales líticos en el registro superficial de la transición Pleistoceno-Holoceno en el macizo del Deseado. En Contra viento y marea: Arqueología de Patagonia, M. T. Civalero, P. Fernández \& A. G. Guraieb, Comps., pp. 71-88. Buenos Aires: Instituto Nacional de Antropología y Pensamiento Latinoamericano.

CAStro de Aguilar, A., 1993. El análisis funcional por medio del estudio microscópico de huellas de uso. Aportes para un modelo de clasificación tipológica. Tesis doctoral de la Facultad de Ciencias Naturales, Universidad Nacional de La Plata, La Plata.

Fiore, D., 1996-1998. Técnicas y artefactos para realizar grabados rupestres. Una investigación bibliográfica. Palimpsesto 5: 208222, Buenos Aires.

— 1999. Cuestiones teórico-metodológicas e implicaciones arqueológicas en la identificación de artefactos utilizados en la producción de grabados rupestres. Hacia una arqueología del arte. Relaciones de la Sociedad Argentina de Antropología XXIV: 277-291, Buenos Aires.

- 2007. The economic site of rock art. Concepts on the production of visual images. Rock Art Research 24 (2): 149-160. Melbourne: AURA.

Fiore, D. \& M. I. Hernández Llosas, 2007. Miradas rupestres: tendencias en la investigación del arte parietal en Argentina. Relaciones de la Sociedad Argentina de Antropología XXXII: 217-242, Buenos Aires.

Gradin, C., 1985. Área de los cazadores meridionales (PampaPatagonia). En Arte rupestre prebistórico de la Argentina, J. Schobinger \& C. Gradin, Eds., pp. 11-49. Milán: Jaca Book.

Gradin, C. \& A. M. Aguerre, 1984. El arte rupestre del Área La Martita. Sección A del Departamento Magallanes, Provincia de Santa Cruz. Relaciones de la Sociedad Argentina de Antropología XV: 195-223, Buenos Aires.

Hermo, D., 2008. Los cambios en la circulación de las materias primas líticas en ambientes mesetarios de Patagonia. Una aproximación para la construcción de los paisajes arqueológicos de las sociedades cazadoras-recolectoras. Tesis doctoral de la Facultad de Ciencias Naturales, Universidad Nacional de La Plata, La Plata.

KeEley, L. H., 1980. Experimental determination of stone tools uses. A microwear analysis. Chicago: University of Chicago Press.

Leipus, M., 2006. Análisis de los modos de uso prehispánicos de las materias primas líticas en el Sudeste de la Región Pampeana. Una aproximación funcional. Tesis doctoral de la Facultad de Ciencias Naturales, Universidad Nacional de La Plata, La Plata. 
Lynch, V.; D. Hermo \& M. Álvarez, 2010. El uso de la forma: análisis funcional del componente inferior de la localidad arqueológica Piedra Museo (Santa Cruz, Argentina). En Libro de resúmenes del V Simposio Internacional "El Hombre Temprano en América: a un siglo del debate Ameghino-Herdlicka", D. Hermo \& R. V. Blanco, Comps., pp. 95-96. Buenos Aires. Cooperativa Gráfica El río suena.

Mansur-Franchomme, M. E., 1983. Traces d'utilisation et technologie lithique: exemples de la Patagonie. Tesis doctoral de la Universidad de Bordeaux I, Bordeaux.

— 1987. El análisis funcional de artefactos líticos. Cuadernos Serie Técnica 1: 1-87. Buenos Aires: Instituto Nacional de Antropología.

Menghin, O. F., 1952. Las pinturas rupestres de la Patagonia. RUNA 5: 5-22, Olavarría.

— 1957. Estilos del arte rupestre de Patagonia. Acta Praehistorica 1: 57-87, Buenos Aires.

Miотті, L., 1991. Manifestaciones rupestres en Santa Cruz: la localidad arqueológica Piedra Museo. En El arte rupestre en la Argentina Contemporánea, M. M. Podestá, M. I. Hernández Llosas \& S. F. Renard de Coquet, Eds., pp. 132-138. Buenos Aires: FECIC.

— 1992. Arqueología experimental: fracturas y marcas óseas. Arqueología Contemporánea 5: 39-64. Buenos Aires: Ediciones Búsqueda.

1994 Ms. Informe I sobre modificaciones de las superficies óseas observadas con MEB: huellas de agentes no antrópicos en arqueofaunas de Pampa y Patagonia. Informe al Consejo Superior de Investigaciones Científicas (CONICET), Buenos Aires.

— 1995. Piedra Museo Locality: a special place in the New World. Current Research in the Pleistocene 12: 36-38, Texas.

— 1996. Piedra Museo, nuevos datos para la ocupación pleistocénica en Patagonia. En Arqueología solo Patagonia, J. Gómez Otero, Ed., pp. 93-101. Puerto Madryn: CENPAT-CONICET.

— 1998. Zooarqueología de la meseta Central y la costa de la provincia de Santa Cruz: Un enfoque de las estrategias adaptativas y los paleoambientes. San Rafael: Museo de Historia Natural de San Rafael.

— 2006. Paisajes domésticos y sagrados desde la arqueología de los cazadores-recolectores en el Macizo del Deseado, provincia de Santa Cruz. Cazadores Recolectores del Cono Sur. Revista de Arqueología 1: 13-42, Buenos Aires.

— 2008. Household and sacred landscapes among Holocene hunter-gatherers of Patagonia's Central Plateau. Before Farming 3: 5-44, London.

MiotTI, L. \& N. CARDEN, 2007. The relationships of rock art and archaeofaunas in the Central Patagonian Plateau. En Taphonomy and Zooarchaeology in Argentina, M. Gutiérrez, L. Miotti, M. Salemme, G. Mengoni \& G. Barrientos, Eds., pp. 203-218. London: British Archaeological Reports (BAR).

Miotti, L. \& R. CATTÁNeO, 1997. Lithic technology at 13000 years ago in southern Patagonia. Current Research in Pleistocene 14: 65-68, Texas.

Miotti, L. \& M. SalEmme, 1999. Biodiversity, taxonomic richness and generalist economical systems in Pampa Patagonia regions, southern South America. Quaternary International 53-54: 53-68, Canadá.
2005. Hunting and butchering events at the Pleistocene/ Holocene transition in Piedra Museo: an example of adaptation strategies of the first colonizers of Patagonia. En Paleoamerican Origins: Beyond Clovis, R. Bonnichsen, Ed., pp. 209-220. Texas: Center for the Study of First Americans and Texas-A\&M University Press.

Miotti, L.; N. Carden \& M. J. Canosa, 1999. Paisajes arqueológicos de cazadores-recolectores, arte rupestre y lagunas. En Actas del XII Congreso Nacional de Arqueología Argentina, C. Diez, Ed., tomo III, pp. 54-63. La Plata: Universidad Nacional de La Plata.

Miotti, L.; N. CARden \& L. Magnin, 2007. Tendencia central, dispersión y orientación en el paisaje regional. Estudios de distribución del arte rupestre en la meseta Central de Santa Cruz. En Arqueología de Fuego-Patagonia. Levantando piedras, desenterrando buesos... y develando arcanos, F. Morello, M. Martinic, A. Prieto \& G. Bahamonde, Eds., pp. 601-612. Punta Arenas: Ediciones CEQUA.

Miotti, L.; D. Hermo, M. Vázquez, N. Carden, M. Giardina \& L. Magnin, 2006. Aguada del Cuero. Un nuevo escalón en la arqueología de la meseta Central de Santa Cruz. En Actas del XIII Congreso Nacional de Arqueología Argentina, E. Berberián, Ed., Vol. 4, pp. 55-62. Córdoba: Universidad de Córdoba.

Miotti, L.; D. Hermo \& N. Carden, 2009. Piedra Museo: una ventana abierta hacia los cazadores-recolectores que colonizaron la meseta Central de Santa Cruz durante finales de la era glaciar. En Estado actual de las investigaciones realizadas sobre Patrimonio Cultural en Santa Cruz, V. Hammar, Coord., pp. 173-184. Río Gallegos: Subsecretaría de Cultura de la provincia de Santa Cruz.

NAMI, H. G., 1991. Algunas reflexiones teóricas sobre arqueología y experimentación. SHINCAL 3 (2): 151-168, Catamarca.

— 2007. Epistemología y consideraciones sobre Arqueología y Tecnología Lítica Experimental, Buenos Aires.

Panza, J. L., 2001. Hoja Geológica 44769-IV. Monumento Natural Bosques Petrificados, Provincia de Santa Cruz, Boletín 258. Buenos Aires: Instituto de Geología y Recursos MineralesServicio Geológico Minero Argentino.

Paunero, R. S., 1992. Manos pintadas en negativo: Un ensayo de experimentación. Revista de Estudios Regionales CEIDER 1: 47-67, San Rafael.

— 1994. Aproximación contextual, pinturas rupestres y experimentación. En Actas y Memorias del XI Congreso Nacional de Arqueología Argentina. Revista del Museo de Historia Natural de San Rafael, Tomo xiII, pp. 98-100, Mendoza.

Semenov, S. A., 1964. Prehistoric technology. London: Cory, Adams \& Mackay.

TRIGGER, B., 1992. Historia del pensamiento arqueológico. Barcelona: Editorial Crítica.

VAUGHAN, P., 1985. Use-wear analysis of flaked stone tools. Arizona: The University of Arizona Press.

ZÁrate, M.; A. Blassi \& J. RABASSA, 2000. Geoarqueología de la localidad Piedra Museo. En Guía de campo de la visita a las localidades arqueológicas. Taller internacional INQUA: La colonización del Sur de América durante la transición Pleistoceno-Holoceno, L. Miotti, R. Paunero, M. Salemme \& R. Cattáneo, Eds., pp. 56-64. La Plata: Imprenta Servicoop. 
